\section{(6) OPEN ACCESS}

\title{
Determinants and outcomes of physical activity in patients with COPD: a systematic review
}

\author{
Elena Gimeno-Santos, ${ }^{1,2,3,4}$ Anja Frei ${ }_{1}^{5,6}$ Claudia Steurer-Stey, ${ }^{6}$ Jordi de Batlle, ${ }^{1,2,7}$ \\ Roberto A Rabinovich, ${ }^{8}$ Yogini Raste, ${ }^{9}$ Nicholas S Hopkinson, ${ }^{9}$ Michael I Polkey, ${ }^{9}$ \\ Hans van Remoortel, ${ }^{10}$ Thierry Troosters, ${ }^{10}$ Karoly Kulich, ${ }^{11}$ Niklas Karlsson, ${ }^{12}$ \\ Milo A Puhan, 5,13 Judith Garcia-Aymerich, 1,2,3 on behalf of PROactive consortium
}

\section{- Additional material is published online only. To view please visit the journal online (http://dx.doi.org/10.1136/ thoraxjnl-2013-204763). \\ For numbered affiliations see end of article. \\ Correspondence to Dr Judith Garcia-Aymerich Centre for Research in Environmental Epidemiology, Doctor Aiguader 88, Barcelona 08003, Spain; jgarcia@creal.cat}

Received 31 October 2013 Revised 24 January 2014 Accepted 29 January 2014 Published Online First 20 February 2014

\section{ABSTRACT}

Background The relationship between physical activity, disease severity, health status and prognosis in patients with COPD has not been systematically assessed. Our aim was to identify and summarise studies assessing associations between physical activity and its determinants and/or outcomes in patients with COPD and to develop a conceptual model for physical activity in COPD.

Methods We conducted a systematic search of four databases (Medline, Embase, CINAHL and Psychinfo) prior to November 2012. Teams of two reviewers independently selected articles, extracted data and used the Grading of Recommendations Assessment, Development, and Evaluation (GRADE) to assess quality of evidence.

Results 86 studies were included: 59 were focused on determinants, 23 on outcomes and 4 on both. Hyperinflation, exercise capacity, dyspnoea, previous exacerbations, gas exchange, systemic inflammation, quality of life and self-efficacy were consistently related to physical activity, but often based on cross-sectional studies and low-quality evidence. Results from studies of pharmacological and non-pharmacological treatments were inconsistent and the quality of evidence was low to very low. As outcomes, COPD exacerbations and mortality were consistently associated with low levels of physical activity based on moderate quality evidence. Physical activity was associated with other outcomes such as dyspnoea, health-related quality of life, exercise capacity and $\mathrm{FEV}_{1}$ but based on cross-sectional studies and low to very low quality evidence.

Conclusions Physical activity level in COPD is consistently associated with mortality and exacerbations, but there is poor evidence about determinants of physical activity, including the impact of treatment.

\section{INTRODUCTION}

A substantial amount of research about physical activity in patients with COPD has been published recently, prompted by studies showing that low levels of physical activity are associated with poor prognosis in COPD $^{1}{ }^{2}$ and by observations that patients with COPD are substantially less physically active than healthy subjects of the same age and sex. ${ }^{3}$ As a result there is the general notion that physical activity is important for patients with COPD.

\section{Key messages}

What is the key question?

- Despite the increasing research on physical activity and COPD, it is unclear which are the determinants and outcomes of physical activity in patients with COPD.

What is the bottom line?

- Physical activity level in COPD is consistently associated with mortality and exacerbations, but this is in contrast to the poor evidence about determinants of physical activity, including current COPD treatments.

Why read on?

- This is a systematic review that will help clinicians to interpret the currently very heterogeneous literature on the topic and investigators to identify which research needs to be prioritised.

Knowledge about the determinants and outcomes of physical activity in patients with COPD is needed to design interventions, to guide further research including randomised clinical trials and to improve the management of patients with COPD. It is currently unclear what the determinants and outcomes of different levels of physical activity are and to what extent current pharmacological and nonpharmacological treatments may modify physical activity levels. A determinant is defined as any factor that brings about change in a health condition or other defined characteristics, such as physical activity levels. ${ }^{5}$ Determinants can be modifiable (eg, dyspnea) or non-modifiable (eg, age), and can also include interventions that may modify levels of physical activity, such as pulmonary rehabilitation or behavioural support. In the same way, an outcome is defined as all the possible results that may stem from an exposure, such as low levels of physical activity. ${ }^{5}$ Occasionally the same variable may be a determinant or an outcome of physical activity (eg, lung function or health related quality of life).

A recent expert review presented an interesting theoretical framework describing the role and consequences of physical inactivity in patients with 
COPD, ${ }^{6}$ but was not based on a systematic and thus a complete review of the available literature of the determinants and outcomes of physical activity. Therefore, our aim was to identify and summarise studies assessing associations between physical activity and its determinants and/or outcomes in patients with COPD.

\section{METHODS}

\section{Data sources and searches}

This study was part of the European Commission funded PROactive project (http://www.proactivecopd.com), which aims to develop and validate patient-reported outcome (PRO) instruments that capture the dimensions of physical activity in daily life relevant to patients with COPD. We utilised standard systematic review methodology following the handbooks of the Centre for Reviews and Dissemination ${ }^{7}$ and the Cochrane Collaboration. ${ }^{8}$ The manuscript follows the PRISMA ${ }^{9}$ statement for reporting of systematic reviews and meta-analyses. All methods were specified in advance, documented in a protocol (see online supplement 1), and approved by the PROactive consortium. Details of the performed searches are provided in online supplement 2 .

The bibliographic details of all retrieved articles were stored in a RefWorks-COS file. We removed duplicate records resulting from the various database searches. The source of identified articles (database, hand search, researcher contacts) was recorded in a 'user defined field' of the RefWorks-COS file. An additional 'user defined field' was assigned to individual reviewers where they recorded their decision for inclusion and exclusion.

\section{Study selection}

Two reviewers independently assessed the title and abstract of every citation retrieved by the database searches (form available in online supplement 3). The decisions of the reviewers (order full text or reject citation) were recorded in the RefWorks-COS file and compared. We ordered all articles that were deemed potentially eligible by at least one member of the consortium. Two independent reviewers evaluated the retrieved full texts and made a decision on inclusion or exclusion according to the predefined selection criteria (form available in online supplement 4). Any disagreements in any phase were resolved by consensus, with close attention paid to the previously defined inclusion/ exclusion criteria. In the case of persistent disagreement, a third member adjudicated. All studies that did not fulfil the predefined criteria were excluded and their bibliographic details were listed with the specific reason for exclusion.

We included studies if they fulfilled the following criteria:

1. Studies in which determinants and outcomes of physical activity, explicitly or implicitly defined as 'any bodily movement produced by skeletal muscles that results in energy expenditure ${ }^{10}$ were assessed.

2. Study design: longitudinal observational studies (prospective and retrospective); randomised and non-randomised trials: both arms (intervention + control) if the outcome was physical activity, or only the control arm when used in an analysis similar to a longitudinal observational study; and crosssectional and case-control observational studies if they included associations with other variables.

3. Population: patients with COPD defined by spirometry (any definition as long as it was based on spirometry).

4. Causal direction (directionality): with directionality we refer to the establishment of a temporal relationship between a determinant (must exist before levels of physical activity change) and physical activity. We included studies in which the design of the study allowed an assumption of causal direction in associations between 'determinant and physical activity' or 'physical activity and outcome', that is, longitudinal studies and clinical trials, and studies in which the design could not address causality, that is, cross-sectional studies, but the authors clearly stated in the introduction or in the methods which was the hypothesised direction.

5. No language or date restrictions were imposed.

\section{Data extraction and quality assessment}

A Microsoft Office Access form was developed and used for data extraction. Two reviewers performed a pilot test of 10 randomly selected articles; the form was then refined prior to the final extraction process. The final version of the data extraction form was used by five independent reviewers to screen the full text of the included studies. Any disagreements were resolved by consensus, with close attention paid to the data extraction criteria (more details in online supplement 5).

We assessed the quality of the evidence for each association of physical activity with its determinants and outcomes following the Grading of Recommendations Assessment, Development and Evaluation (GRADE) approach ${ }^{11}{ }^{12}$ (more details in online supplement 5).

\section{Data synthesis and analysis}

The results of the data extraction were summarised in structured tables, one for determinants and one for outcomes. We did not perform meta-analyses because we deemed the studies to be too different to summarise their results statistically. However, we illustrated the individual effect of each study graphically for those determinants and/or outcomes that showed consistent results (eg, all showing results in the same direction) and when the quality of evidence was at least moderate. We developed a conceptual model based on the determinants and outcomes included in the assessment and on the quality of evidence, highlighting the directionality, the consistency and the confidence rating.

\section{RESULTS}

\section{Summary of studies}

Figure 1 shows the flow diagram of the identification of the studies, from 3687 references to 86 articles that were finally included. Online supplementary table S1 shows the reasons for exclusion. From 86 studies included, 59 assessed only determinants of physical activity in COPD, 23 only outcomes and 4 both. Table 1 shows information on reference details, study design and number of subjects included in each study (S1-S86 on references section of online supplement). Regarding the study design, 36 were cross-sectional, 4 case-control, 16 longitudinal, 12 randomised controlled trials and 18 non-randomised and/or non-controlled studies. Online supplementary table S2 shows detailed information on patients' characteristics and instruments for measuring physical activity of the 86 studies included. Almost half of the studies $(n=38)$ measured physical activity using only a direct method (accelerometer and/or pedometer), 43 used only an indirect method (validated or nonvalidated questionnaire), and 5 used both direct and indirect methods.

A large body of research focused on typical COPD characteristics as determinants of physical activity, such as $\mathrm{FEV}_{1}(13$ studies), exercise capacity measured by 6 -min walk distance or $\mathrm{VO}_{2}$ peak obtained during an incremental exercise test (7 studies) and dyspnoea (6 studies). 


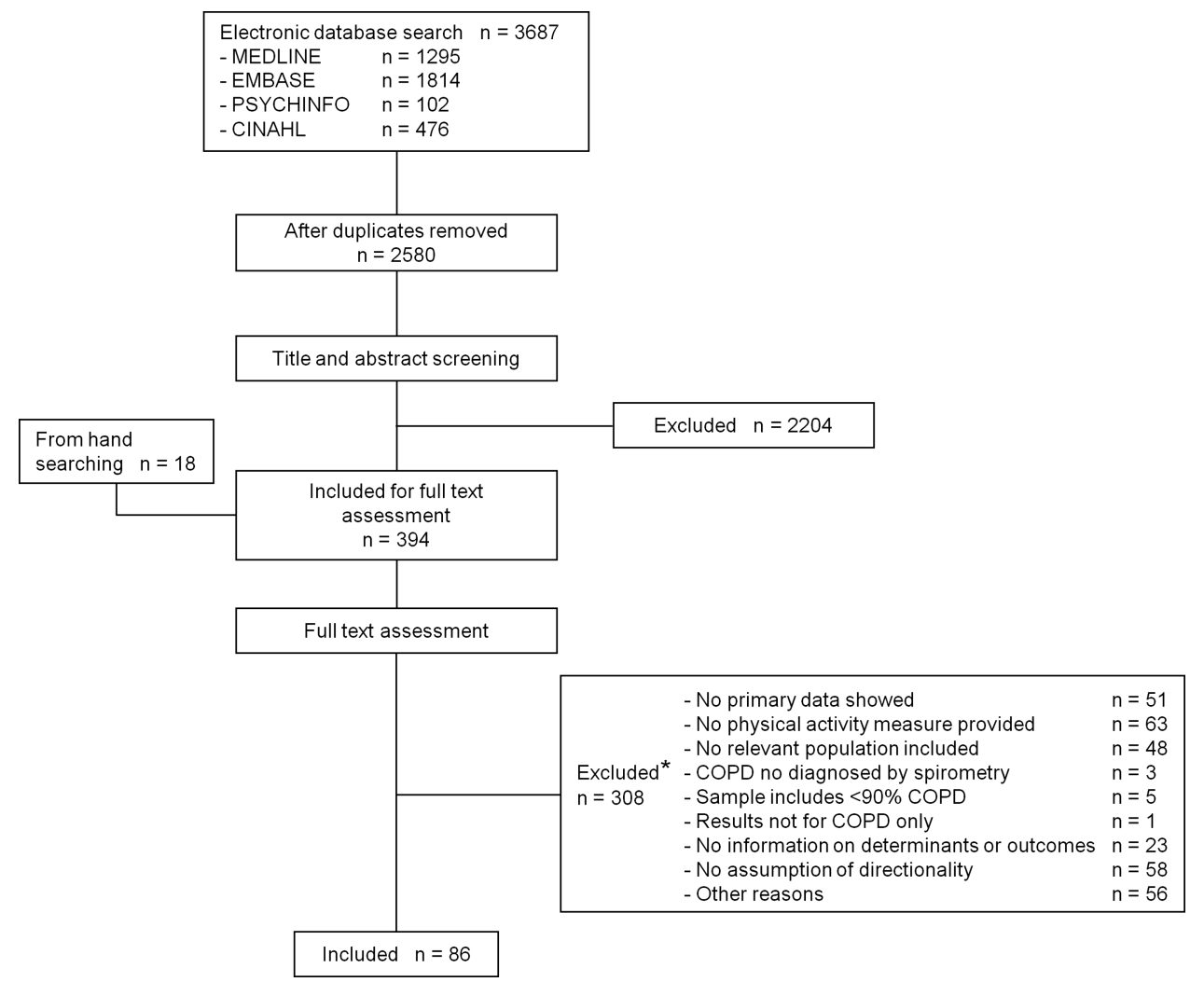

Figure 1 Flow diagram of process of systematic literature search. *Details for reason for exclusion in online supplementary table S1.

\section{Socio-demographic, lifestyle and environmental determinants}

Between two and six studies assessed the role of sex, age, cultural group, marital status, socioeconomic status, education, working status, smoking status, alcohol consumption and day of the week as potential determinants of physical activity in patients with COPD. In most of the studies the design was cross sectional, thus limiting interpretation of causal direction. In addition, most studies did not adjust observed associations for potential confounders. Finally, for all of these potential determinants, the results were not consistent across studies. For example, current smoking status compared with former was statistically associated with higher physical activity levels in a given study, ${ }^{\mathrm{S} 33}$ while no statistically significant differences in physical activity were found between smoking groups in another study. ${ }^{\mathrm{S} 34}$ Detailed information on estimates of associations, statistically significant and non-significant, is provided in online supplementary table S3. Overall, the quality of evidence for sex and age as determinants of physical activity in patients with COPD was moderate and very low for the remaining sociodemographic, lifestyle and environmental factors (table 2A).

\section{Clinical and functional determinants}

Regarding clinical and functional determinants, including $\mathrm{FEV}_{1}$, FVC, hyperinflation, gas exchange, exercise capacity, body mass index (BMI), dyspnoea, systemic inflammation, comorbidities, previous exacerbations, quality of life, self-efficacy or emotional status, most studies were cross sectional and lacked adjustment for confounders. Associations of physical activity with hyperinflation, gas exchange, exercise capacity, dyspnoea, systemic inflammation, previous exacerbations, quality of life and selfefficacy were consistent, but were inconsistent for $\mathrm{FEV}_{1}, \mathrm{FVC}$, BMI, comorbidities and emotional status. Overall, almost all associations were based on evidence graded as very low quality, except the association with hyperinflation which was graded as low quality (table $2 \mathrm{~B}$, details in online supplementary table S3).

\section{Drug and non-drug treatments}

A large body of literature exists on the effect of exercise training on physical activity (21 studies). Although most studies were clinical trials so that the direction of the association could be established, some of them did not include a control group or included a control group without randomised allocation. Some did not control for confounders in the analysis. Furthermore, results on the effects of these interventions were inconsistent and the quality of evidence was low. Other non-pharmacological interventions identified in this review include physical activity advice, long-term oxygen therapy and dietary interventions. Again, design and analytical limitations preclude confidence on the results, so the quality of the evidence was rated as low to very low. Finally, only three studies tested the effect of pharmacological treatment (long-acting 32 agonist and/or oral and inhaled corticosteroids) on physical activity and combine observational with experimental designs. The results were inconsistent and have several methodological flaws, so the quality of this evidence was rated as very low (table $2 \mathrm{C}$, details in online supplementary table S3).

\section{Outcomes}

The literature covering the effect of physical activity on outcomes in COPD was smaller than for the determinants of physical activity, but the overall quality of evidence was better. Clinically relevant outcomes such as COPD exacerbations and mortality were assessed in eight and seven studies, respectively, which were of longitudinal design (thus showing the outcome to occur subsequent to a level of physical activity being 
Table 1 Reference details, design and number of subjects of 86 studies reporting associations between physical activity and its determinants or outcomes in patients with COPD

\begin{tabular}{|c|c|c|c|c|c|c|c|c|}
\hline Reference & Study design & $\mathrm{n}$ & Reference & Study design & $\mathbf{n}$ & Reference & Study design & n \\
\hline Altenburg WA, 2013 $3^{\mathrm{S1}}$ & Cross sectional & 155 & Garcia-Aymerich J, 2004 ${ }^{530}$ & Cross sectional & 346 & Pitta, F. $2009^{559}$ & Cross-sectional & 80 \\
\hline Beauchamp MK, $2012^{\mathrm{S2}}$ & Cross sectional & 37 & Garcia-Aymerich J, 2006 ${ }^{\mathrm{S} 31}$ & Cohort & 2386 & Pomidori, L. $2012^{560}$ & Randomised non-controlled parallel study & 36 \\
\hline Behnke $\mathrm{M}, 2005^{53}$ & Non-randomised controlled study & 88 & Garcia-Aymerich J, $2008^{532}$ & Cohort & 2226 & Probst, VS. $2011^{561}$ & Randomised non-controlled parallel study & 40 \\
\hline Bendstrup KE, $1997^{54}$ & Randomised controlled trial & 32 & Garcia-Aymerich J, $2009^{533}$ & Cross sectional & 341 & Roig, M. $2011^{\mathrm{S} 62}$ & Cohort & 101 \\
\hline Benzo $R, 2010^{55}$ & Cohort & 597 & Garcia-Rio F, $2009^{534}$ & Cross sectional & 110 & Sandland, CJ. $2008^{563}$ & Randomised controlled trial & 20 \\
\hline Berry M, $2006^{56}$ & Cross sectional & 291 & Garcia-Rio F, $2012^{535}$ & Cohort & 173 & Schou, L. $2013^{564}$ & Randomised controlled trial & 44 \\
\hline Berry $\mathrm{M}, 2010^{57}$ & $\begin{array}{l}\text { Randomised non-controlled } \\
\text { parallel study }\end{array}$ & 176 & Goto $Y, 2004^{536}$ & $\begin{array}{l}\text { Non-randomised } \\
\text { controlled study }\end{array}$ & 30 & Sewell, L. $2005^{565}$ & Randomised non-controlled parallel study & 180 \\
\hline Bestall J, $1999^{58}$ & Cross sectional & 100 & Hartman JE, $2013^{537}$ & Cross sectional & 113 & Sewell, L. $2010^{566}$ & Non-controlled study & 95 \\
\hline Bon J, $2011^{59}$ & Cross sectional & 190 & Hataji $0,2013^{538}$ & Non-controlled study & 23 & Silva, DR. $2011^{567}$ & Cross-sectional & 95 \\
\hline Bossenbroek L, $2009^{510}$ & Case-control & 62 & Inal-Ince D, $2005^{539}$ & Cross sectional & 30 & Skumlien, S. $2006^{568}$ & Cross-sectional & 110 \\
\hline Bourbeau J, $2007^{511}$ & Cohort & 421 & Jehn $\mathrm{M}, 2012^{540}$ & Cross sectional & 107 & Skumlien, S. $2008^{569}$ & $\begin{array}{l}\text { Non-randomised non-controlled } \\
\text { parallel study }\end{array}$ & 40 \\
\hline Breyer MK, $2010^{512}$ & Randomised controlled trial & 60 & Katajisto $\mathrm{M}, 2012^{\mathrm{S41}}$ & Cross sectional & 719 & Takigawa, N. $2007^{570}$ & Non-controlled study & 225 \\
\hline Chao PW, $2011^{513}$ & Cross sectional & 21 & Lahaije $A, 2013^{542}$ & Cross sectional & 57 & Troosters, T. 2010, Respir Med ${ }^{571}$ & Cross-sectional & 70 \\
\hline Chen Y, $2006^{514}$ & Cohort & 145 & Lee $\mathrm{H}, 2011^{\mathrm{S43}}$ & Cross sectional & 131 & Tsara, V. $2008^{572}$ & Case-control & 133 \\
\hline Coronado $\mathrm{M}, 2003^{\mathrm{S} 15}$ & Non-controlled study & 15 & Lemmens KMM, $2008^{544}$ & Cross sectional & 278 & Van Gestel, AJ. $2012^{573}$ & Cross-sectional & 154 \\
\hline Dal Negro R, $2010^{516}$ & Randomised controlled trial & 32 & Lore, $\mathrm{V}, 2006^{545}$ & Cross sectional & 23 & Van Remoortel, H. $2013^{574}$ & Cross-sectional & 59 \\
\hline Dallas MI, $2009^{517}$ & Non-controlled study & 45 & Mador MJ, $2011^{546}$ & Non-controlled study & 24 & Vergeret, J. $1989^{575}$ & Cohort & 243 \\
\hline Daly C, $2011^{\mathrm{S18}}$ & Non-controlled study & 8 & Miravitlles M, $2011^{547}$ & Cohort & 346 & Waatevik, M. $2012^{576}$ & Cross-sectional & 370 \\
\hline de Blok BM, $2006^{519}$ & Randomised controlled trial & 21 & Monteiro F, $2012^{548}$ & Cross sectional & 74 & Wakabayashi, R. $2011^{577}$ & Randomised controlled trial & 102 \\
\hline Effing T, 2011 $11^{520}$ & Randomised controlled trial & 153 & Moy M, $2009^{549}$ & Cross sectional & 17 & Wakabayashi, R. $2011^{578}$ & Cross-sectional & 389 \\
\hline Egan C, $2012^{521}$ & Non-controlled study & 47 & Moy M, $2013^{550}$ & Cohort & 169 & Walker, PP. $2008^{579}$ & Non-controlled study & 23 \\
\hline Eisner MD, $2008^{522}$ & Cross-sectional & 1202 & Nguyen HQ, $2009^{551}$ & Randomised controlled trial & 17 & Waschki, B. $2011^{580}$ & Cohort & 169 \\
\hline Eliason $\mathrm{G}, 2011^{523}$ & Cross-sectional & 44 & Nguyen HQ, $2013^{552}$ & Cross-sectional & 148 & Watz, H. $2008^{581}$ & Cross-sectional & 170 \\
\hline Esteban C, $2006^{524}$ & Cohort & 611 & Nield $\mathrm{M}, 2005^{553}$ & Non-controlled study & 48 & Watz, H. $2009^{582}$ & Cross-sectional & 170 \\
\hline Esteban C, $2010^{525}$ & Cohort & 391 & Okubadejo AA, $1997^{554}$ & Case-control & 42 & Watz, H. $2009^{583}$ & Cross-sectional & 163 \\
\hline Esteban C, $2011^{\text {S26 }}$ & Cohort & 611 & Palop Cervera M, $2010^{555}$ & Case-control & 125 & Weekes, CE. $2009^{584}$ & Randomised controlled trial & 59 \\
\hline Faager $\mathrm{G}, 2004^{527}$ & Randomised controlled trial & 20 & Pitta F, $2006^{556}$ & Cohort & 17 & Wewel, A. $2008^{585}$ & Non-controlled study & 21 \\
\hline Faulkner J, $2010^{528}$ & Randomised controlled trial & 20 & Pitta F, 2006 557 & Cross sectional & 23 & Yeo, J. $2006^{586}$ & Cross-sectional & 27 \\
\hline Garcia-Aymerich J, $2003^{529}$ & Cohort & 340 & Pitta F, $2008^{558}$ & Cross sectional & 40 & & & \\
\hline
\end{tabular}


Table 2 Quality of evidence for determinants and outcomes of physical activity in COPD, as identified in 86 studies

Determinan

N studies Direction established Control for confounding Directness Consistency Strength Low precision Other

Confidence rating

(A) Quality of evidence for socio-demographic, lifestyle and environmental determinants of physical activity

Age $^{530} 533534557571581$

Alcohol consumption 530534

Cultural group ${ }^{514} 559571$

Day of the week 54554982

Education $530 \mathrm{S33} 534$

Marital status ${ }^{530} 533$

Sex 530533534557568581

Smoking habit ${ }^{530} 533534$

Socioeconomic status ${ }^{\mathrm{S30}} \mathrm{533}$

Working status 530533534

(B) Quality of evidence for functional and clinical determinants of physical activity

BODE index 534549557581583

Body mass index ${ }^{530} 534548557581$

Cardiovascular ${ }^{530} 581$

Dyspnoea $^{88541} 530534552557583$

Emotional status ${ }^{530} 552$

Exercise capacity $\left(\mathrm{VO}_{2}\right.$ max, 6MWD) ${ }^{51} \mathrm{56} 534537549552557574583$

$\mathrm{FEVV}_{1}^{56} 522530534541542549557558571581582583586$

FVC $534 \$ 57$

Gas exchange (DLco $)^{534574}$

Gas exchange $\left(\mathrm{PCO}_{2}\right)^{\mathrm{S30}} \mathrm{534}$

Gas exchange $\left(\mathrm{PO}_{2}\right)^{\mathrm{S30}} \mathrm{S34}$

Hyperinflation $^{534} 537542557558$

Osteoarticular condition ${ }^{530} 549$

Previous exacerbation ${ }^{511} 530556$

Quality of life/health-related quality of life ${ }^{51} 530 \$ 34 \$ 49557$

Self-efficacy 537

Systemic inflammation ${ }^{534} 581$

(C) Quality of evidence for interventions to modify levels of physical activity

Dietary intervention 516584

Exercise training ${ }^{33} 5457512515517518520521527528546553560561564565566569570579$

Long-acting $\beta 2$ agonist/corticosteroids $530 \$ 34 \$ 33$

Long-term oxygen therapy 530534554563572575

Physical activity advice ${ }^{519} 551585$

Outcome

(D) Quality of evidence for outcomes as a result of different levels of physical activity

Balance $^{52562}$

Bone mineral density ${ }^{59} 567$

Dyspnoea $^{533544}$

Exacerbations $^{55} \$ 14 \$ 29 \varsigma 31532535550 \$ 56$
$-2^{2}$

$-1^{2}$

$-2^{2}$

$-2^{2}$
$-2^{2}$

$-2^{2}$

$-2^{2}$

na

$-1^{4}$

$-1^{4}$

$-1^{4}$

$-1^{4}$

na

$-1^{4}$
$-1^{5}$

$-1^{4}$

$-2^{2}$

$-2^{2}$

$-2^{2}$

$-2^{2}$

$-2^{2}$

$-2^{2}$

$-1^{2}$

$-1^{2}$
$-2^{2}$

$-2^{2}$

$-2^{2}$

$-2^{2}$

$-2^{2}$

$-1^{2}$

$-2^{2}$

$-2^{2}$

$-2^{2}$

Yes

Yes

Yes

\begin{tabular}{|c|c|c|c|c|c|}
\hline Yes & $-1^{1}$ & No & No & No & +++ (moderate) \\
\hline Yes & $-1^{1}$ & No & $-1^{6}$ & No & + (very low) \\
\hline$-1^{3}$ & $-1^{1}$ & No & No & No & + (very low) \\
\hline Yes & $-1^{1}$ & No & No & No & + (very low) \\
\hline Yes & $-1^{1}$ & No & No & No & + (very low) \\
\hline Yes & $-1^{1}$ & No & No & No & + (very low) \\
\hline Yes & $-1^{1}$ & No & No & No & +++ (moderate) \\
\hline Yes & $-1^{1}$ & No & $-1^{6}$ & No & + (very low) \\
\hline Yes & $-1^{1}$ & No & No & No & + (very low) \\
\hline Yes & $-1^{1}$ & No & No & No & + (very low) \\
\hline Yes & $-1^{1}$ & No & No & No & + (very low) \\
\hline Yes & $-1^{1}$ & No & No & No & + (very low) \\
\hline Yes & $-1^{1}$ & No & $-1^{6}$ & $-1^{7}$ & + (very low) \\
\hline Yes & yes & No & No & No & + (very low) \\
\hline Yes & $-1^{1}$ & No & No & No & + (very low) \\
\hline Yes & yes & No & No & No & + (very low) \\
\hline Yes & $-1^{1}$ & No & No & No & + (very low) \\
\hline Yes & $-1^{1}$ & No & No & $-1^{12}$ & + (very low) \\
\hline Yes & yes & No & No & No & + (very low) \\
\hline Yes & yes & No & No & No & + (very low) \\
\hline Yes & yes & No & No & No & + (very low) \\
\hline Yes & yes & No & No & No & ++ (low) \\
\hline Yes & $-1^{1}$ & No & $-1^{6}$ & No & + (very low) \\
\hline Yes & yes & No & No & $-1^{7}$ & + (very low) \\
\hline Yes & yes & No & No & No & + (very low) \\
\hline Yes & yes & No & No & $-1^{7}$ & + (very low) \\
\hline Yes & yes & No & No & $-1^{7}$ & + (very low) \\
\hline Yes & $-1^{1}$ & No & No & $-1^{12}$ & ++ (low) \\
\hline Yes & $-1^{1}$ & No & No & No & ++ (low) \\
\hline Yes & $-1^{1}$ & No & $-1^{6}$ & No & + (very low) \\
\hline Yes & $-1^{1}$ & No & No & $-1^{10,11}$ & ++ (low) \\
\hline Yes & $-1^{1}$ & No & $-1^{9}$ & No & + (very low) \\
\hline
\end{tabular}




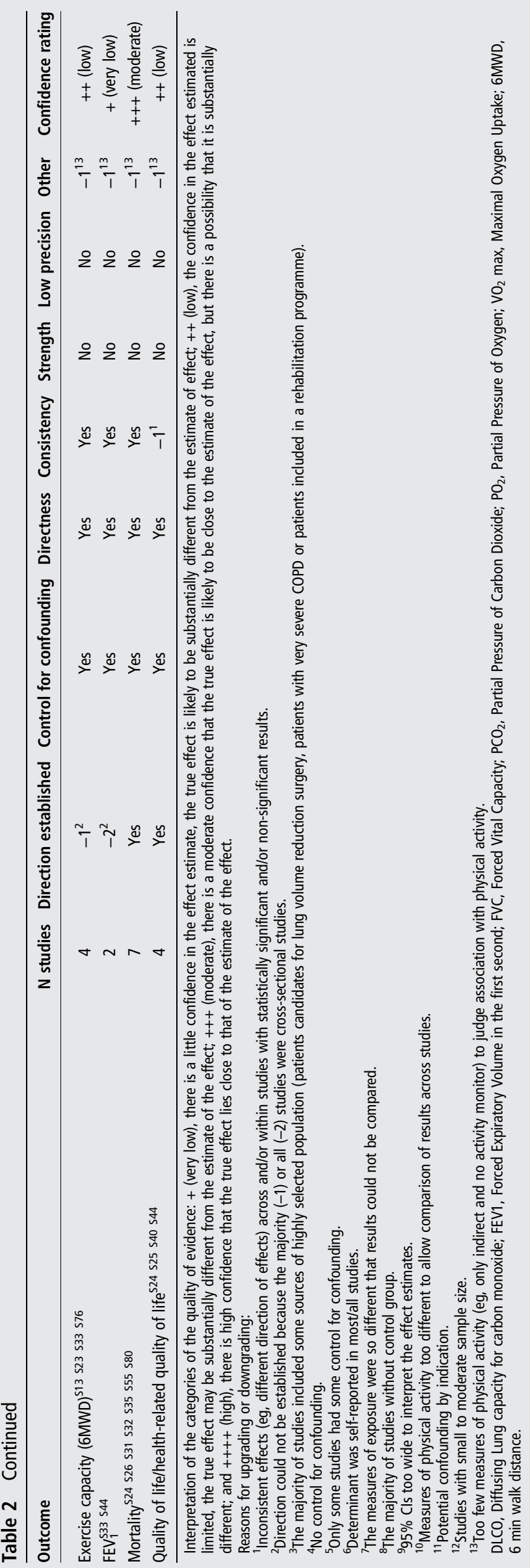

defined), controlled for confounders and provided consistent results. Therefore, quality of evidence was moderate for both outcomes. Unfortunately, we could not perform any meta-analysis because of the heterogeneity of the studies but we illustrated graphically the effect estimate in each study for exacerbations (figure 2) and mortality (figure 3). Associations for other clinical outcomes such as dyspnoea, health-related quality of life, exercise capacity and $\mathrm{FEV}_{1}$ were lacking in consistency. Although the underlying studies are often of high quality, we downgraded the quality of evidence from high to moderate because of a lack of direct measures of physical activity. Therefore, quality of evidence is rated as low to very low (table 2D, details in online supplementary table S4).

\section{Conceptual model for physical activity in patients with COPD}

Using information from table 2, we developed a conceptual model for physical activity in COPD (figure 4). In addition to quality of the evidence, the figure also provides visual information on causal direction and the consistency of associations as supported by existing literature.

\section{DISCUSSION}

The main findings of this systematic review are, first, there are many studies on clinical, functional, socio-demographic and lifestyle factors as determinants of physical activity in patients with COPD. Unfortunately, the quality of evidence remains very low, mainly because the causal direction of these associations could not be established due to the cross-sectional nature of most studies or because of a lack of control for confounding factors. Second, only a few interventions have been tested as determinants of physical activity, all with inconsistent results. Third, there is consistent evidence of effects of physical activity on COPD exacerbations and mortality.

\section{Significance of the findings}

This systematic review provides important information on what we know today and where research needs to be prioritised. First, available information today does not clearly answer what the determinants of physical activity are in patients with COPD. This is mostly due to the fact that available research has been cross sectional, which even in the case of consistent results, does not conclusively establish if a factor needs to be considered a determinant (exists before levels of physical activity change) or an outcome (results of different levels of physical activity). Some may argue that the rich available data on determinants of exercise capacity (eg, hyperinflation, ventilatory response to exercise, muscle weakness or dyspnoea) ${ }^{13-15}$ can be extrapolated as determinants of physical activity. However, it is well known that physical activity (a complex behaviour) and capacity or physical fitness (a set of attributes) are correlated only to a moderate extent ${ }^{10}$ and constitute different concepts. This is supported by the fact that some determinants of exercise capacity are not associated with physical activity.

Second, evidence for the effect of therapeutic interventions on physical activity levels is of low to very low quality and is inconsistent. The most studied intervention is exercise training, with 21 studies assessing the effects of exercise training on physical activity in patients with COPD. However, some limitations lowered the confidence in the estimates of effect, such as the lack of a control group in half of the studies or the inconsistency of results (eg, half of studies found significant associations and half did not). In addition, it is important to point out that we included all these interventions that offered at least exercise 
Figure 2 Graphical illustration of the individual study effects of physical activity on reduced risk of exacerbations. Pitta 2006 was excluded from the graph because it did not provide a risk ratio.

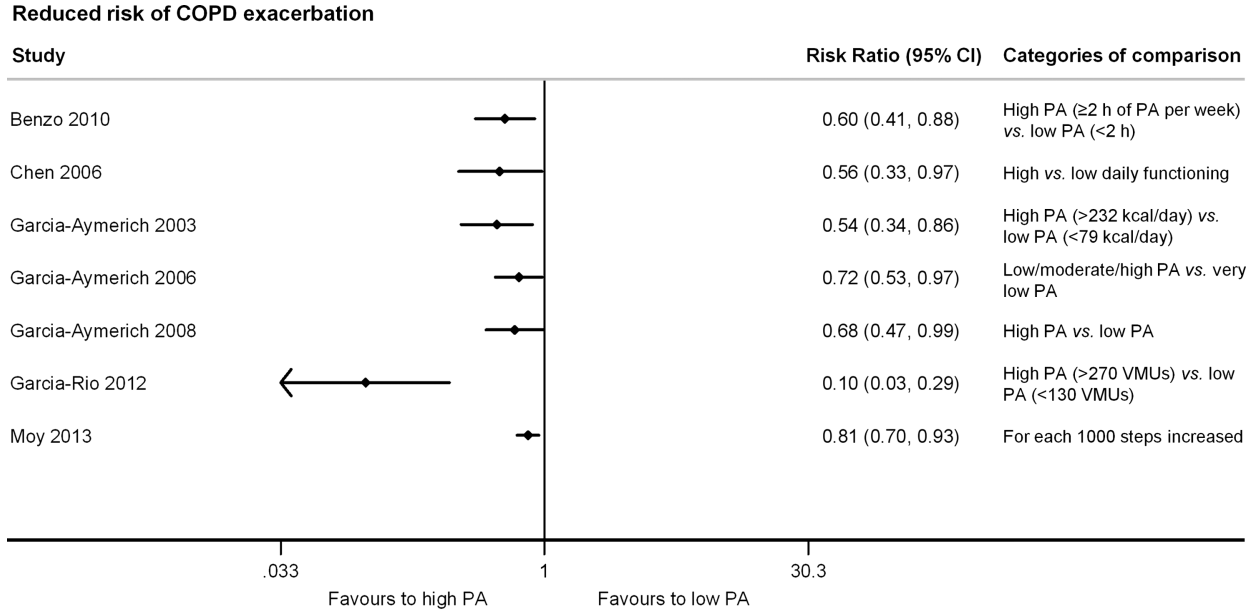

training but we did not consider the type of exercise, the duration of the intervention programme or the training intensity used, which varied significantly across studies. Surprisingly, there is scarce evidence about the effects of commonly used pharmacological agents, such as long-acting $\$ 2$ agonists, on physical activity levels. This should attract attention of the industry since some drug treatments could theoretically increase levels of physical activity, for example, as a result of decreased hyperinflation, reduced symptoms and enhanced exercise endurance.

Third, we identified that the best available evidence is for the association of physical activity with mortality and COPD exacerbations. These results are based on longitudinal studies, the results are consistent, and all estimates of associations were adjusted for known confounders, such as age, sex, BMI, severity and previous exacerbations. Nevertheless, the quality of the evidence is still moderate since physical activity was assessed using questionnaires instead of more precise tools, such as activity monitors in the largest studies. Finally, we could not establish some clinically important parameters such as $\mathrm{FEV}_{1}$, dyspnoea and exercise capacity as clear outcomes of physical activity because of the lack of consistent results across studies, small sample sizes leading to imprecise estimates and cross-sectional study design (not allowing directionality). Indeed, diverse crosssectional studies approached the associations between physical activity and the above-mentioned parameters, some under the hypothesis they would be determinants of physical activity, while other authors hypothesised they would be outcomes of physical activity. Several studies in the general population support bi-directionality for these associations. ${ }^{16} 17$

\section{Critique of the method}

A strength of this review is the rigorous systematic review methodology followed which was key to dealing effectively with a very heterogeneous literature. Additionally, we report significant and non-significant associations, which allows a comprehensive evaluation of the consistency and strength of the associations described. A challenge of this review was to identify whether a specific association should be categorised as 'determinant', 'outcome' or be excluded. In most cross-sectional studies the authors did not clearly state the hypotheses about the directionality of associations. Our selection of some (but not all) crosssectional studies and non-directional measures of association (such as correlation coefficients) was restricted to those where the authors clearly described the hypothesis about directionality in the introduction or methods section.

\section{Future research direction}

The results of the present review should be taken into account for further research. Specifically, physical activity should be included as an outcome in randomised controlled trials to provide knowledge on the potential effects of drug and

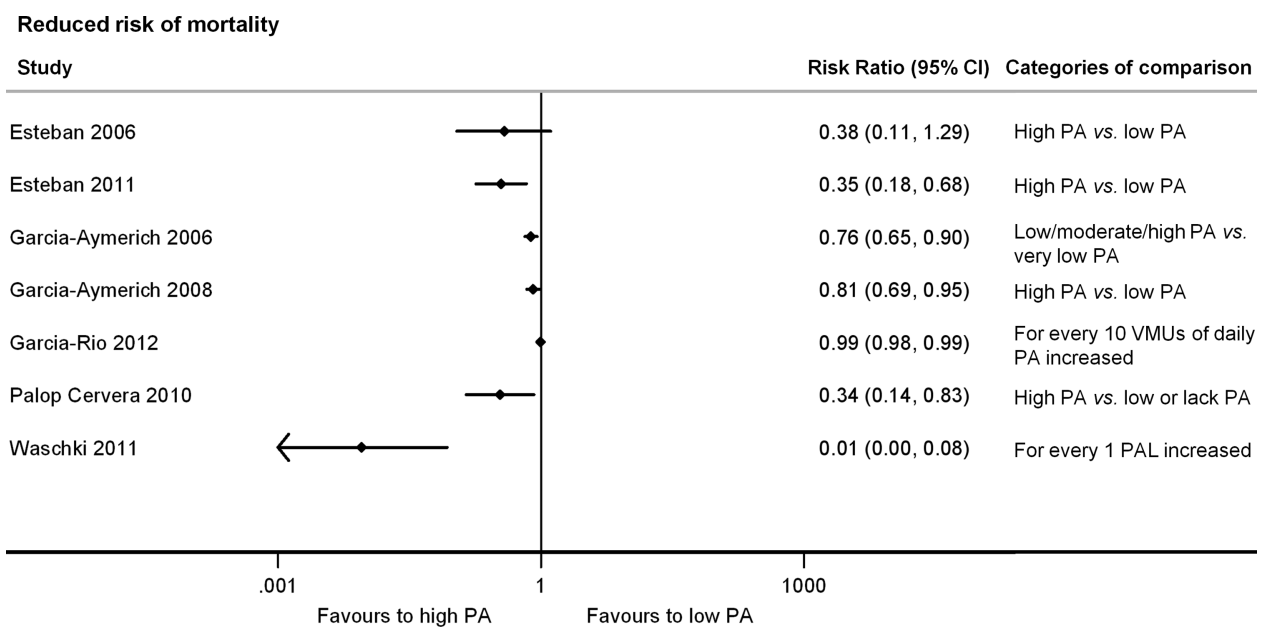

Figure 3 Graphical illustration of the individual study effects of physical activity on reduced risk of mortality. 
Figure 4 Conceptual model of physical activity in patients with COPD. Grading was done separately for each individual determinant/outcome (table 2). Variables of the same category sharing the same confidence rating are grouped together in this figure for clarity. Socio-demographic, lifestyle and environment, and some clinical variables $\left(\mathrm{FEV}_{1}\right.$, body mass index, emotional status and comorbidities) do not show consistent effects on physical activity. The association between other clinical and functional determinants and physical activity such as hyperinflation, exercise capacity, dyspnoea, previous exacerbations, gas exchange, systemic inflammation, quality of life and self-efficacy is consistent across studies, but lacking directionality because mostly it is based on cross-sectional studies. Studies on pharmacological and non-pharmacological treatments as determinants of physical activity are all longitudinal and thus, by design, provided a basis for a clear direction of the associations. But the results are inconsistent with some treatments showing an increase in physical activity and some showing no effect.

Regarding the outcomes, only COPD exacerbation and mortality show consistent effects with clear directionality and based on moderate quality evidence.

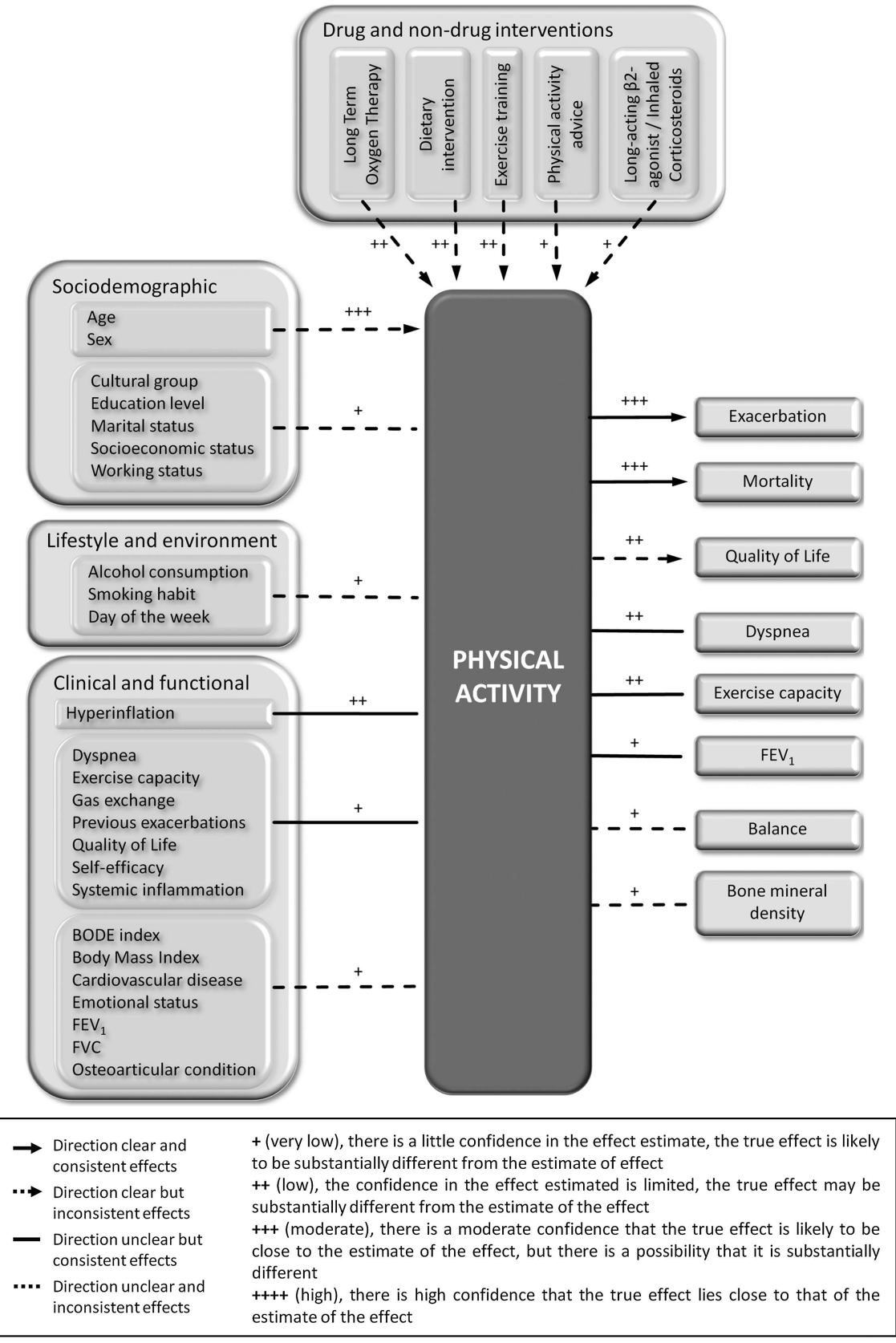

non-drug treatments on this relevant outcome. Also, this review supports the need, prior to start any study, of a careful review of the existing literature in order not to miss (in data collection or statistical analyses) relevant potential confounders. We acknowledge that cross-sectional studies are easier to perform than longitudinal studies but the quality assessment here shows that they can just yield low-quality evidence about the association of determinants and outcomes of physical activity. The research community should make an effort to conduct prospectively planned cohort studies and randomised trials to provide a stronger evidence base for determinants and outcomes of physical activity and for developing recommendations for or against treatments in clinical guidelines.

In conclusion, the consistent effects of physical activity on mortality and COPD exacerbation are in contrast to the poor evidence about determinants of physical activity, including current COPD treatments and other outcomes of physical activity. There is a need for high-quality observational studies and randomised trials that use valid and accurate measurements of physical activity.

\section{Author affiliations}

${ }^{1}$ Center for Research in Environmental Epidemiology (CREAL), Barcelona, Spain

${ }^{2}$ CIBER Epidemiología y Salud Pública (CIBERESP), Barcelona, Spain

${ }^{3}$ Universitat Pompeu Fabra (UPF), Barcelona, Spain

${ }^{4}$ FCS Blanquerna, Research Group in Physiotherapy (GReFis), Universitat Ramon Llull, Barcelona, Spain

${ }^{5}$ Institute of Social and Preventive Medicine, University of Zurich, Zurich, Switzerland

${ }^{6}$ Institute of General Practice and Health Services Research, University of Zurich,

Zurich, Switzerland

${ }^{7}$ Section of Nutrition and Metabolism, International Agency for Research on Cancer, Lyon, France

${ }^{8}$ ELEGI/Colt Laboratory, UoE/MRC Centre for Inflammation Research, University of Edinburgh, Edinburgh, UK

${ }^{9}$ NIHR Respiratory Biomedical Research Unit of the Royal Brompton and Harefield NHS Foundation Trust and Imperial College London, London, UK

${ }^{10}$ Respiratory Division, Katholieke University of Leuven, Leuven, Belgium

${ }^{11}$ Respiratory Department, Novartis, Basel, Switzerland

${ }^{12}$ R\&D, AstraZeneca, Södertälje, Sweden 
${ }^{13}$ Department of Epidemiology, Johns Hopkins Bloomberg School of Public Health, Johns Hopkins University, Baltimore, Maryland, USA

Acknowledgements The authors thank Mr Jordi Figuerola (Centre for Research in Environmental Epidemiology) for creating the Microsoft Office Access data extraction form and Dr Marla K Beauchamp, Dr Michael Berry, Dr Dina Brooks, Dr Francisco Garcia-Rio, Dr Yoko Goto, Dr Fabio Pitta, Dr Vanessa S Probst and Dr Benjamin Waschki for sending the data requested from their manuscripts.

Contributors MAP and JG-A led the systematic review. EG-S, AF, RAR, NSH, MIP, $\mathrm{HvR}, \mathrm{TT}, \mathrm{KK}, \mathrm{NK}, \mathrm{MAP}$ and JG-A developed the study protocol. EG-S and AF conducted the electronic database searches; EG-S conducted the additional searches. EG-S and AF coordinated the references in RefWorks. EG-S, AF, YR, NSH, MIP, HVR, KK, MAP and JG-A screened titles and abstracts. EG-S, AF, CS-S, YR, NSH, MIP, HvR, MAP and JG-A assessed full texts. EG-S, AF, CS-S, JdB, MAP and JG-A extracted the relevant data. EG-S, AF, MAP and JG-A performed the quality grading. EG-S, MAP and JG-A drafted the manuscript. All authors contributed to revising the manuscript and approved the final version. PROactive consortium approved the final version of the manuscript.

Funding The PROactive project is funded by the European Commission Innovative Medicines Initiative Joint Undertaking (IMI JU \# 115011).

Competing interests MIP institution received payment $(<\$ 5000)$ for the participation in an advisory board organised by Boehringer Ingelheim. TT provided consultancy or given sponsored talks around the topic to Boehringer Ingelheim, Novartis and GSK (amounts paid to the institution $<5000 €$ ). KK is employed by Novartis. NK is employed by AstraZeneca.

Provenance and peer review Not commissioned; externally peer reviewed.

Open Access This is an Open Access article distributed in accordance with the Creative Commons Attribution Non Commercial (CC BY-NC 3.0) license, which permits others to distribute, remix, adapt, build upon this work non-commercially, and license their derivative works on different terms, provided the original work is properly cited and the use is non-commercial. See: http://creativecommons.org/ licenses/by-nc/3.0/

\section{REFERENCES}

1 Garcia-Aymerich J, Lange P, Benet $\mathrm{M}$, et al. Regular physical activity reduces hospital admission and mortality in chronic obstructive pulmonary disease: a population based cohort study. Thorax 2006;61:772-8.
2 Garcia-Aymerich J, Lange P, Serra I, et al. Time-dependent confounding in the study of the effects of regular physical activity in chronic obstructive pulmonary disease: an application of the marginal structural model. Ann Epidemiol 2008:18:775-83.

3 Pitta F, Troosters T, Spruit MA, et al. Characteristics of physical activities in daily life in chronic obstructive pulmonary disease. Am J Respir Crit Care Med 2005; 171:972-7.

4 Troosters T, Sciurba F, Battaglia S, et al. Physical inactivity in patients with COPD, a controlled multi-center pilot-study. Respir Med 2010;104:1005-11.

5 Centers for Disease Control and Prevention website. Resource Library: Glossary of Epidemiology Terms. http://www.cdc.gov/excite/library/glossary.htm\#D (accessed 8 Jan 2014).

6 Hartman JE, Boezen HM, de Greef MH, et al. Consequences of physical inactivity in chronic obstructive pulmonary disease. Expert Rev Respir Med 2010;4:735-45.

7 Centre for Reviews and Dissemination Systematic reviews. CRD's guidance for undertaking reviews in health care. York: University of York, 2009. http://www.york. ac.uk/inst/crd/SysRev/!SSL!/WebHelp/SysRev3.htm

8 Higgins JPT, Green S. Cochrane Handbook for Systematic Reviews of Interventions, version 5.0.2 (update February 2009).

9 Liberati A, Altman DG, Tetzlaff J, et al. The PRISMA statement for reporting systematic reviews and meta-analyses of studies that evaluate health care interventions: explanation and elaboration. Ann Intern Med 2009;151:W65-94.

10 Caspersen CJ, Powell KE, Christenson GM. Physical activity, exercise, and physical fitness: definitions and distinctions for health-related research. Public Health Rep 1985; 100:126-31.

11 Guyatt GH, Oxman AD, Schunemann HJ, et al. GRADE guidelines: a new series of articles in the Journal of Clinical Epidemiology. I Clin Epidemiol 2011;64:380-2.

12 Schunemann H, Hill S, Guyatt G, et al. The GRADE approach and Bradford Hill's criteria for causation. J Epidemiol Community Health 2011;65:392-5.

13 Bauerle 0, Chrusch CA, Younes M. Mechanisms by which COPD affects exercise tolerance. Am J Respir Crit Care Med 1998;157:57-68.

14 Foglio K, Carone M, Pagani M, et al. Physiological and symptom determinants of exercise performance in patients with chronic airway obstruction. Respir Med 2000;94:256-63.

15 Gosselink R, Troosters T, Decramer M. Peripheral muscle weakness contributes to exercise limitation in COPD. Am J Respir Crit Care Med 1996;153:976-80.

16 Garcia-Aymerich J, Lange P, Benet M, et al. Regular physical activity modifies smoking-related lung function decline and reduces risk of chronic obstructive pulmonary disease: a population-based cohort study. Am J Respir Crit Care Med 2007:175:458-63.

17 Spirduso WW, Cronin DL. Exercise dose-response effects on quality of life and independent living in older adults. Med Sci Sports Exerc 2001;33:S598-608. 


\section{Correction}

Gimeno-Santos E, Frei A, Steurer-Stey C, et al. Determinants and outcomes of physical activity in patients with COPD: a systematic review. Thorax 2014;69:731-39. doi:10.1136/ thoraxjnl-2013-204763

The following Collaborator list should be included in this article.

PROactive consortium: Chiesi Farmaceutici S.A.: Caterina Brindicci, Tim Higenbottam; Katholieke Universiteit Leuven: Thierry Trooster, Fabienne Dobbels; Glaxo Smith Kline: Margaret X. Tabberer; University of Edinburgh, Old College South Bridge: Roberto Rabinovich, Bill McNee; Thorax Research Foundation, Athens: Ioannis Vogiatzis; Royal Brompton and Harefield NHS Foundation Trust: Michael Polkey, Nick Hopkinson; Municipal Institute of Medical Research, Barcelona: Judith Garcia-Aymerich; Universität Zürich, Zürich: Milo Puhan, Anja Frei; University Medical Center, Groningen: Thys van der Molen, Corina De Jong; Netherlands Asthma Foundation, Leusden: Pim de Boer; British Lung Foundation, UK: Ian Jarrod; Choice Healthcare Solution, UK: Paul McBride; European Respiratory Society, Lausanne: Nadia Kamel; Pfizer: Katja Rudell, Frederick J Wilson; Almirall: Nathalie Ivanoff; Novartis: Karoly Kulich, Alistair Glendenning; AstraZeneca AB: Niklas X Karlsson, Solange Corriol-Rohou; UCB: Enkeleida Nikai; Boehringer Ingelheim: Damijen Erzen.

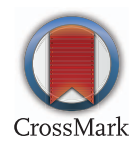

Thorax 2014;69:810. doi:10.1136/thoraxjnl-2013-204763corr1 